\title{
On the Masses and on the Mass Transfer \\ in the Interactive Binary SS 433
}

\author{
TOMAŽ ZWITTER \\ University of Ljubljana, Dept. of Physics, Ljubljana, Slovenia
}
SANDRO D'ODORICO AND TOM OOSTERLOO
European Southern Observatory, Garching, Germany
and

MASSIMO CALVANI

Astronomical Observatory, Padua, Italy

\begin{abstract}
Recent spectroscopic observations of the interactive binary SS 433 with the ESO's New Technology Telescope show that the compact object is a neutron star. We note that the huge mass transfer rate (which may well be the reason for the uniqueness of SS 433) suggests that a significant fraction of the mass lost from the normal star actually settles on the neutron star. This may have important consequences for the structure of the accretion disk and for the formation of jets in this peculiar system.
\end{abstract}

The interactive binary system SS 433 is known for its relativistic jets (Abell \& Margon 1979), its precessional motions of the accretion disk (Wagner 1986), as well as for its mass outflows along the jets and around the disk's plane (Zwitter et al. 1991). All these features are believed to be a consequence of a run-away mass transfer regime making SS 433 the only binary star with super-Eddington luminosity (Calvani \& Nobili 1983).

The mass of the compact object is an important parameter of any model for the binary system as well as for the formation, acceleration and collimation of the jets. Neutron stars differ from black holes not only for their mass but also for the influence on their surroundings: a hard surface and a (rotating) magnetic field of a neutron star influences the motion of the matter coming from the accretion disk in a qualitatively different way than in the case of a black hole. A neutron star can also provide a nearly perfect precessional clock in the system (Begelman \& Rees 1984).

SS 433 is a single lined spectroscopic eclipsing binary. So the mass of the compact object $\left(M_{x}\right)$ and of the mass losing star $\left(M_{*}\right)$ can be estimated from the mass function

$$
F=1.43 \times 10^{-6}\left(K / \mathrm{kms}^{-1}\right)^{3} \mathrm{M}_{\odot}=\frac{M_{*}}{(1+q)^{2}}=\frac{M_{x}}{q(1+q)^{2}}
$$

using the Doppler amplitude $K$ of the corresponding line and the mass ratio $q$ (estimated from the duration and shape of the eclipses). In the case of SS 433 the line to be used is the He II emission line at $4686 \AA$ as it is the only line with the 
phasing compatible with photometric eclipses (Crampton \& Hutchings 1981). The mass ratio $q \equiv M_{x} / M_{*} \approx 0.25$ (Zwitter 1990) was estimated from the length of the $\mathrm{X}$-ray eclipses. The advantage of using the $\mathrm{X}$-rays comes from the fact that a bulk of them comes from a small region around the jet funnels (Watson et al. 1986) making this small region a kind of a "lighthouse" moving around the orbit. The situation in the optical is much more involved as the light we see originates not only from the two stars, but also from the accretion disk, its corona and the complicated inflow and outflow streams (Zwitter et al. 1991).

The amplitude of the He II line at $4686 \AA$ was originally measured by Crampton \& Hutchings (1981). They claimed a rather high Doppler amplitude $K \sim$ $195 \mathrm{~km} \mathrm{~s}^{-1}$ corresponding to $F \sim 10 \mathrm{M}_{\odot}$. This strongly suggested a black hole $\left(M_{x} \approx 4 \mathrm{M}_{\odot}\right)$ nature of the compact object (cf. eq. 1). However the uncertainties in the estimates of both $q$ and $K$ are large. A rapidly rotating mass losing star would have the same size as a much heavier corotating star, thus it would push up the estimate of the mass ratio $q$ (based on the length of eclipses) considerably. The uncertainties of the $K$ amplitude of the He II line were even larger: the profiles of the line as measured by Crampton \& Hutchings were very complicated, un-accurately sampled and of low amplitude. As a result large systematic errors in their $K$ velocity cannot be excluded.

Recently new observations of the He II line region were obtained (D'Odorico et al. 1991) with the EMMI spectrograph on the New Technology Telescope of the European Southern Observatory. The spectra obtained are of much higher quality than in earlier studies: a resolution of $0.9 \AA$ and a $\mathrm{S} / \mathrm{N}$ ratio of $\approx 30 / 1$ (per $0.3 \AA$ wavelength bin in the line) were achieved. As a consequence the line is resolved and the orbital motion of the whole line can be measured.

The whole profile of the line was found to be moving with the orbital cycle with an amplitude of $K=112 \pm 5 \mathrm{~km} \mathrm{~s}^{-1}$. This corresponds to the mass function $F \simeq 2 \mathrm{M}_{\odot}$ and in turn (using $q \approx 0.25$ ) to the mass of the compact object $M_{x} \simeq$ $0.8 \mathrm{M}_{\odot}$. This is too low for a black hole, so the compact object should be a neutron star. The uncertainties in the estimates of the mass ratio can be responsible for the unusually low value of the mass of the neutron star.

This important result has some relevant consequences for the accretion regime in the vicinity of the compact object. Neutron star has a hard surface so the matter which is accreted should finally settle on it. This is different from a black hole where the matter should only pass the horizon. It is interesting to note that the fraction of the mass leaving the mass-losing star which should be accreted to the neutron star can be estimated. The argument is based on the evolution of the binary system. The rate of mass loss from the loser is huge, estimated at $\dot{M}_{*}=10^{-4}-$ $10^{-5} \mathrm{M}_{\odot} \mathrm{yr}^{-1}$ (Margon and Anderson 1989). Given the uniqueness of SS 433 on the sky the expansion of the mass losing star on the thermal time-scale is unlikely to be the only cause for a big mass loss rate. The mass loss should be at least partly caused also by a shrinking of the Roche lobe of the mass-loser. Such a run-away mass transfer is effective if the separation $a$ of the stars' centers shrinks as well. The time derivative of the orbital separation is proportional to 


$$
\dot{a} \propto \frac{q(1+2 q)-(2+q)\left|\dot{M}_{x} / \dot{M}_{*}\right|}{1+q}
$$

where $\dot{M}_{*}$ is the mass loss rate from the mass-losing star and $\dot{M}_{x}$ is the part of it which is accreted to the compact object. The above equation can be easily derived from the Kepler law assuming the conservation of the orbital angular momentum of the system.

The time derivative in eq. 2 is negative if at least $17 \%$ of the mass lost from the mass losing star is actually accreted to the neutron star. For $q>0.25$ (i.e. more massive compact object) this fraction is even higher and, in our view, imposes significant constraints on the supercritical accretion regime in the vicinity of the compact object.

To summarize, the mass of the compact object in SS 433 favours it to be a neutron star and not a black hole. The mass-losing star fills its Roche lobe which should be shrinking with time. As a consequence at least $17 \%$ of the mass lost from the normal star should not be blown away in jets and other outflows but should be accreted to the neutron star. If the supercritical regime around the compact object can be such that the majority of mass flow actually settles on the neutron star, SS 433 will finally coalesce; this seems a plausible end of evolution for such a unique binary system.

\section{References}

Abell, G. O., and Margon, B. 1979, Nature, 279, 701.

Begelman, M. C., and Rees, M. J. 1984, Mon. Not. R. Astr. Soc., 206, 209.

Calvani, M., and Nobili, L. 1983, in Astrophysical Jets, A. Ferrari and K. Pacholczyk (Eds.), (Dordrecht: Reidel).

Crampton, D., and Hutchings, J. B. 1981, Astrophys. J., 251, 604.

D'Odorico, S., Oosterloo, T., Zwitter, T., and Calvani M. 1991, Nature, 353, 329. Margon, B., and Anderson, S. F. 1989, Astrophys. J., 347, 448.

Watson, M. G., Stewart, G. C., Brinkmann, W., and King, A. R. 1986, Mon. Not. R. Astr. Soc., 222, 261.

Wagner, R. M. 1986, Astrophys. J., 308, 152.

Zwitter, T. 1990, PhD Thesis, International School for Advanced Studies, Trieste, Italy.

Zwitter, T., D‘Odorico, S., Oosterloo, T., and Calvani, M. 1991, Astr. Astrophys., (in print). 